\title{
Dissipative cryogenic filters with zero DC resistance
}

\author{
Hendrik Bluhm* and Kathryn A. Moler \\ Departments of Physics and Applied Physics, Stanford University, Stanford, CA 94305
}

\begin{abstract}
The authors designed, implemented and tested cryogenic RF filters with zero DC resistance, based on wires with a superconducting core inside a resistive sheath. The superconducting core allows low frequency currents to pass with negligible dissipation. Signals above the cutoff frequency are dissipated in the resistive part due to their small skin depth. The filters consist of twisted wire pairs shielded with copper tape. Above approximately $1 \mathrm{GHz}$, the attenuation is exponential in $\sqrt{\omega}$, as typical for skin depth based RF filters. By using additional capacitors of $10 \mathrm{nF}$ per line, an attenuation of at least $45 \mathrm{~dB}$ above $10 \mathrm{MHz}$ can be obtained. Thus, one single filter stage kept at mixing chamber temperature in a dilution refrigerator is sufficient to attenuate room temperature black body radiation to levels corresponding to $10 \mathrm{mK}$ above about $10 \mathrm{MHz}$.
\end{abstract}

\section{INTRODUCTION}

Many cryogenic experiments involve electronic transport measurements, which typically require leads connecting the sample to room temperature instrumentation. Without appropriate filtering, those electrical connections will also transmit parasitic signals to the sample. Many samples are very sensitive to such disturbances, especially when the relevant energy scales are in the milliKelvin region and the devices are small. The effect of extrinsic noise, which can be either anthropogenic or thermal from higher temperature sources, can simply be to heat the electrons in the sample above the lattice temperature. Because of their small volume, mesoscopic devices are particularly sensitive to even very small amounts of power. ${ }^{1}$ In some experiments, such as SET-based devices or solid state qubits, microwave radiation may directly affect the quantum dynamics under study through effects like photon assisted tunneling ${ }^{2}$ or excess dephasing. ${ }^{3}$

To mitigate these undesirable effects, it is crucial that electrical connections to the sample are filtered sufficiently. ${ }^{4}$ With an increasing number of experimentalists exploring quantum physics at the nano scale, there is a growing need for simple filtering solutions. We present here a filter design similar to the one in Ref. 5, but with a DC resistance limited only by contact resistances. It allows us to supply currents on the order of $50 \mathrm{~mA}$ to the mixing chamber of a dilution refrigerator while maintaining a respectable base temperature. In our case, such large currents are required in order to locally apply a magnetic field using a micron scale field coil integrated in a scanning SQUID susceptometer ${ }^{6}$, intended primarily for magnetic response measurements on mesoscopic samples. ${ }^{7,8}$ Similar requirements also occur for flux bias lines of superconducting qubits. Most flux biased qubit experiments currently employ a conventional superconducting magnet, but the individual tuning of several qubits in a well shielded environment ${ }^{9}$ is only achievable with locally generated fields.

The vanishing DC resistance of our filters is achieved by using composite wires with a superconducting core surrounded by resistive material, which are wrapped in copper tape to form a lossy transmission line. At DC, all the current is flowing in the superconducting core, so that there is no dissipation. At higher frequencies, the skin effect restricts the current flow to the resistive part, so that once the skin depth $\delta$ is smaller than the thickness of the resistive sheath, the dissipation quickly approaches that of a purely resistive conductor. The low frequency attenuation is increased by adding two discrete capacitors to ground along each line. At frequencies below the intrinsic inverse $R C$ time of the transmission line, those capacitors together with the frequency-dependent resistance of the transmission line act as a 2-pole $R C$ filter. When they become ineffective above their self resonance, the attenuation is entirely due to the transmission line. Since some of our wires can tolerate a larger resistance, we have also implemented filters with purely resistive wires, which allowed us to reduce their length.

Our filters provide an attenuation of at least $45 \mathrm{~dB}$ above $10 \mathrm{MHz}$ and reach $120 \mathrm{~dB}$ at $500 \mathrm{MHz}$, with a strong extrapolated increase at higher frequencies. This attenuation exceeds the ratio of the photonic power spectral density $S(\omega, T)=\hbar \omega /\left(\exp \left(\hbar \omega / k_{B} T\right)-1\right)$ at room temperature to that at $10 \mathrm{mK}$ above $f=\omega / 2 \pi=10$ $\mathrm{MHz}$ (See fig. 4). Thus, it is sufficient to use a single filter stage. Since dissipative circuit elements, including filters, emit thermal radiation, they have to be kept at the base temperature of the cryostat. Filters for nine resistive and six superconducting twisted wire pairs inside the copper tape shield fit into a cylindrical space with a diameter of $80 \mathrm{~mm}$ and a height of $120 \mathrm{~mm}$. If required, additional filtering to eliminate extrinsic radiation can be added at room temperature, for example at a shielded room feedthrough or cryostat breakout box.

The remainder of this paper is organized as follows: After briefly describing filtering concepts that have appeared in the literature so far in section II, we discuss the physical implementation of our design in section III. A model for the filter is developed in section IV, followed by test result in section $\mathrm{V}$. 


\section{CONVENTIONAL FILTERING STRATEGIES}

This section will give a brief overview of different filtering strategies suitable for cryogenic applications. A more detailed review can be found in Ref. 4. Commercially available reflective filters such as pi-filters are a convenient choice for filtering technical noise at room temperature. However, impedance mismatches in the connected lines may cause resonances which severely compromise their performance, and combining several stages to achieve a high attenuation may not have the desired result. If they are to be used at cryogenic temperatures, one should keep in mind that the properties of some models may change dramatically at low $T$.

Simple $R C$ filters may be quite useful for low to intermediate frequencies if a large resistance (and/or capacitance) can be tolerated. At microwave frequencies, parasitic inductances and capacitances of the lumped elements tend to limit their performance, in contrast to copper powder or transmission line filters which typically feature a very strong attentions above about $1 \mathrm{GHz}$. Copper powder filters are the classic design for low temperature applications, first reported in Ref. 10 and further optimized in reference Ref. 11. They are based on the dissipation of energy by high frequency stray fields through eddy currents. Since copper powder filters are usually made with a single wire, they are more convenient for coaxial cables than for twisted pairs. Due to the high cutoff frequency (e.g. $15 \mathrm{~dB}$ at $100 \mathrm{MHz}),{ }^{4}$ a combination with an $R C$ or $L C$ stage is often necessary. Ref. 12 reports a variant with superconducting wire, which shows a similar attenuation as our filters if configured as pi-filters.

Over the last decade, various versions of dissipative transmission lines have been used as filters. Some of them are essentially resistive coaxial cables. ${ }^{13}$ Vion et al. ${ }^{14}$ have developed a relatively compact lithographic implementation. More recently, the Schoelkopf group reported a very flexible and low cost version based on resistive wire wrapped in copper tape, ${ }^{5}$ which underlies the approach described here. Our modifications of this design are primarily the use of wire with a superconducting core, which eliminates the undesired dc resistance, and the addition of discrete capacitors for increased attenuation at intermediate frequencies. With the exception of Vion's compact filters, the above transmission line filters rely on the skin effect, which confines $\mathrm{AC}$ magnetic fields and therefore currents in a conductor with resistivity $\rho$ to one skin depths $\delta=\sqrt{2 \rho / \mu_{0} \omega}$ below the surface. Due to this constriction of the current flow, the resistance increases as $\sqrt{\omega}$, leading to an attenuation of the form $\exp \left(-\sqrt{\omega / \omega_{0}}\right)$.

\section{PHYSICAL IMPLEMENTATION}

The transmission line part of our filters consists of "copper tape worms" as described in Ref. 5. Three twisted pairs are arranged in parallel and wrapped in copper tape, which forms the outer conductor of the transmission line and shields the signal-carrying wires. Our first tape worm generation was sealed by soldering along the seams as indicated in Fig. 1 in order to achieve optimal RF tightness. However, the soldering process appeared to degrade the wire insulation, which caused frequent shorts to the copper tape or between the wires. Furthermore, unavoidable thickness variations of the solder seam lead to sharp kinks at the thinnest spots which sometimes caused the copper tape to break. To avoid these problems, we eliminated the soldering step in a second generation. To maintain an acceptable RF tightness without soldering, it is crucial to have a large overlap along the seams. The width of the unsoldered filter ribbon $(\approx 8 \mathrm{~mm})$ was about one third of that of the tape used, so that the overlap extended over the full ribbon width. The test results reported in Sec. V are from a prototype filter of the first generation.

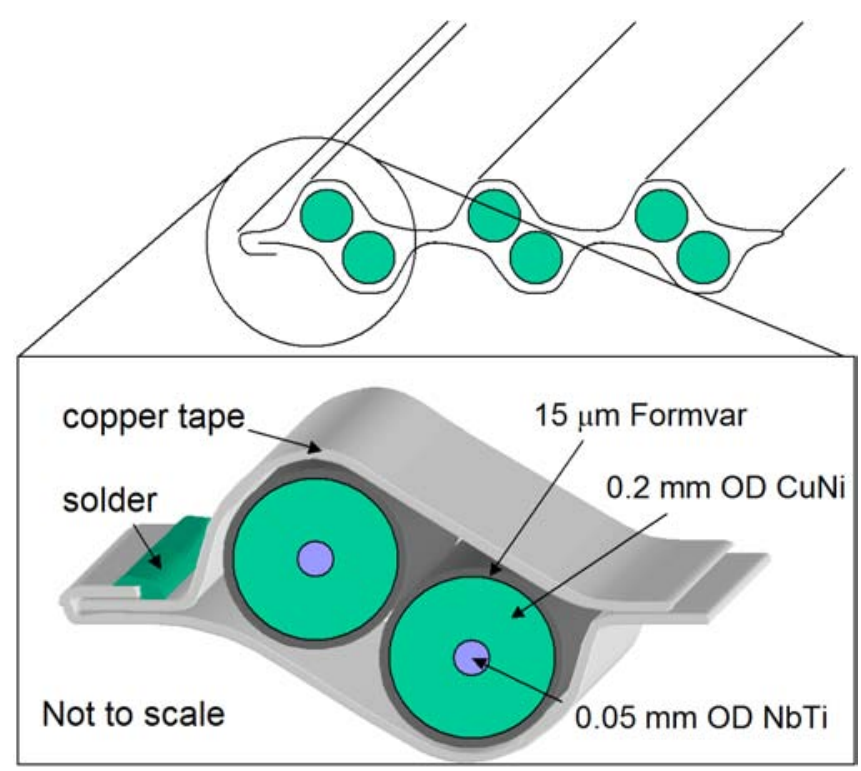

FIG. 1: (color online) Schematic cross section of a copper tape worm of the first generation, with a soldered seam. The width of a ribbon with three twisted pairs is about $8 \mathrm{~mm}$.

We used 3M copper tape with non-conductive adhesive (type 1194). The conductive-adhesive variety (1181) is unsuitable because its adhesive contains small metal spikes that can penetrate the wire insulation. Since even some tape rolls with nominally non-conductive adhesive tend to be contaminated with such spikes, the tape should be checked carefully under a microscope and discarded if any spikes appear.

The wire used for the superconducting filters is a customized version of persistent current switch wire purchased from Supercon Inc. The $0.05 \mathrm{~mm}$ diameter superconducting core is made of $\mathrm{NbTi}$ and the resistive outer part of $\mathrm{Cu}_{70} \mathrm{Ni}_{30}$, with a resistivity of $38.5 \mu \Omega \mathrm{cm}$. To obtain a sufficient resistance at $10 \mathrm{MHz}$, we chose a 
total outer wire diameter of $0.2 \mathrm{~mm}$. The wire insulation is $15 \mu \mathrm{m}$ thick Formvar. The non-superconducting filters were made of 37 AWG Stablohm 800 wire, which has a resistivity of $132 \mu \Omega \mathrm{cm}$. Their insulation has a thickness of $6 \mu \mathrm{m}$ and $16 \mu \mathrm{m}$ for the first and second generation, respectively. The thinner insulation was chosen initially to maximize the capacitance between the wire and the tape, but then replaced in order to reduce the likelihood of shorts.

To increase the attenuation between about 10 and 100 $\mathrm{MHz}$, we connected $5.1 \mathrm{nF}$ capacitors form the 700B series manufactured by American Technical Ceramics Inc. (ATC) between each filter wire and the shield, one quarter of the total filter length from each end. Those high quality RF capacitors are very compact (approximately $2.8 \mathrm{~mm}$ cubes) and have a high self resonance of $100 \mathrm{MHz}$ and a negligible temperature dependence. They were placed in $20 \mathrm{~mm}$ long boxes made of $3 / 16$ " square brass profile. The ends of these filter housings were closed by soldering in matching brass plugs with a hole to feed through the wires. One contact of the capacitors was directly glued onto those plugs using Epotek $\mathrm{H} 20 \mathrm{E}$ conductive epoxy. In the first generation, the copper tape shield was soldered directly into the holes of the end plugs. These direct connections were replaced by short adapter pieces made of $1 / 16$ " brass tubing for improved mechanical stability in the second generation. In order to protect the wire from excessive heating and sharp metal corners, the part inside the brass tubing was threaded through Teflon tubing. The same techniques were used to connect the tape worm to copper parts used as feedthroughs towards the sample area. To avoid vacuum problems, the capacitor boxes were filled with Stycast 1266 through small holes, which were later electrically sealed with silver epoxy.

The superconducting filters are about $4 \mathrm{~m}$ long. Due to their larger resistance, $2 \mathrm{~m}$ are enough for the resistive version. They were accommodated in our dilution refrigerator by coiling them around an OFHC copper support below the mixing chamber. For $2 \mathrm{~m}$ of filter ribbon with three twisted pairs, this requires a height of about 1.5 $\mathrm{cm}$ and a diameter of about $8 \mathrm{~cm}$, including the space for the capacitors. Fig. 2 shows a partially assembled superconducting filter consisting of two such coils. For good thermal contacts, the filters were clamped between machined copper parts with groves to accommodate the wires. It is important not to apply pressure to the latter to avoid shorts.

After elimination of initial problems with frequent shorts, the filters turned out to be reliable over several cooldowns. Some of them were operated at voltages of more than $\pm 180 \mathrm{~V}$ with respect to ground, i.e. $360 \mathrm{~V}$ between the wires of a pair. However, they tend to suffer from dunking in liquid nitrogen, as some liquid enters the tape worm and forces it apart when expanding upon warming up.

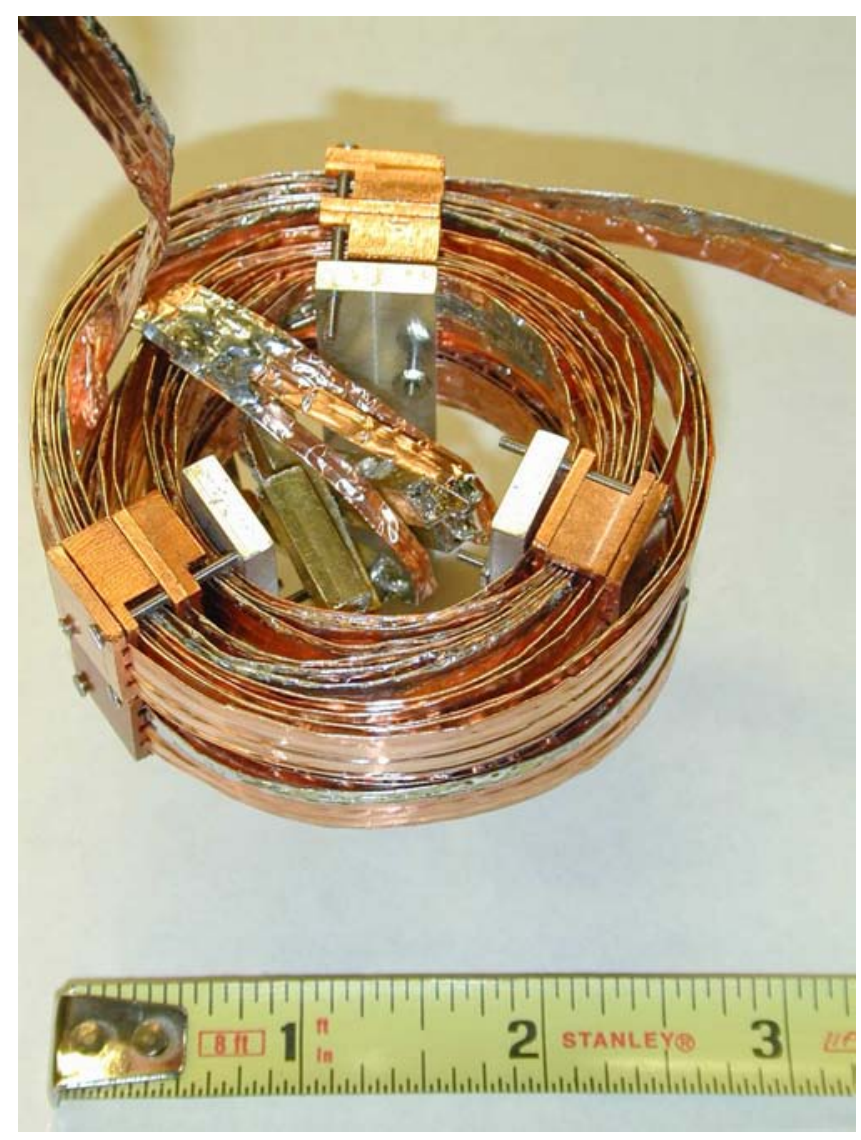

FIG. 2: (color online) Partially assembled superconducting filter. When fully assembled, several such filters are stacked and the aluminum bars shown in the picture are replaced by long copper bars connecting the filters. The brass parts in the center accommodate the capacitors.

\section{MODELING}

\section{A. Approximate analysis}

Although the model discussed in section IV B provides a fairly accurate quantitative description, the simplified analysis of this section is useful in order to better understand the significance of the different model parameters and as a rough tool for preliminary design work. In the following, $C$ and $L$ denote the capacitance and inductance per unit length of the transmission line.

The dissipation in the resistive material becomes significant once the skin depth $\delta=\sqrt{2 \rho / \mu_{0} \omega}$ becomes of order or smaller than the thickness $r_{i}-r_{c}$, where $r_{i}$ and $r_{c}$ are the radius if the inner conductor and its superconducting core, respectively. Above this cutoff, the current flows within a layer of thickness $\delta$ below the surface, so that the frequency dependent resistance per unit length is $R(\omega) \approx \rho / 2 \pi r_{i} \delta=\left(1 / 2 \pi r_{i}\right) \sqrt{\omega \rho \mu_{0} / 2}$.

At frequencies where the discrete capacitors are dominant, their time constant $R(\omega) C$ needs to be as large 
as possible for strong attenuation. The value of the capacitors is limited by availability and size considerations. $R(\omega)$ is at the first glance proportional to $\sqrt{\rho}$, however the wire thickness $2 r_{i}$ required to force the current into the resistive sheath also increases as $\sqrt{\rho}$, so that not much would be gained from a higher resistivity.

For the chosen type of wire, the cutoff frequency according to the above criterion is $\rho / \pi \mu_{0}\left(r_{i}-r_{c}\right)^{2}=17$ $\mathrm{MHz}$. The resistance at cutoff is about $\rho / \pi r_{i}^{2}=12 \Omega / \mathrm{m}$, giving an $R C$ roll off frequency of $1.3 \mathrm{MHz}$ for a $2 \mathrm{~m}$ long section terminated by a $5 \mathrm{nF}$ capacitor. Thus, one can expect a significant attenuation at cutoff. (Note that the resistance at $1.3 \mathrm{MHz}$ is much smaller though.)

For a 2-pole $R C$ filter with $R \propto \sqrt{\omega}$, one obtains a roll off of $60 \mathrm{~dB} /$ decade. This will provide sufficient attenuation up to the capacitor self resonance at $100 \mathrm{MHz}$. At larger frequencies, the transmission line needs to provide all the attenuation. We first derive a simple expression for its performance.

In a transmission line with perfect conductors and a homogeneous dielectric with relative permittivity $\epsilon_{r}$, the relation between the electric and magnetic fields of a TEM mode ${ }^{15}$ imply that $L C=\epsilon_{r} / c^{2}$, with $c$ denoting the speed of light in vacuum. Thus, the line impedance $Z_{0}=\sqrt{L / C}$ is given by $Z_{0}=\sqrt{\epsilon_{r}} / C c{ }^{17}$ With $I, P$ and $V$ denoting the current, power and voltage of a forward propagating mode in the line, the power loss per unit length is $d P / d x=R I^{2}=R I V / Z_{0}=\left(R / Z_{0}\right) P$. Integrating this along the transmission line gives a power attenuation of $e^{-l R / Z_{0}}$, where $l$ is the total length. Since $Z_{0} \propto 1 / C$ as explained above, we thus have to maximize the $R C$ constant of the line. As the capacitance is proportional to the area over which the wire faces the shield and $R$ is the surface impedance divided by the wire circumference, the $R C$ product is approximately independent of the wire diameter. However, $R(\omega) \propto \sqrt{\rho}$, so that a high resistivity is desirable, as long as $r_{i}-r_{c}$ is adapted accordingly.

\section{B. Model for a coaxial geometry}

We now turn to the more accurate model that we used to fit the test results. The copper tape worm sections are modeled as a dissipative transmission line with a frequency dependent resistance per unit length, $R(\omega)$. According to the standard transmission line $\operatorname{model}^{16}$, the local voltage between the two conductors, $V(x)$, and the longitudinal current, $I(x)$, are related by $V(x)=$ $V_{0} e^{ \pm \gamma(\omega) x}$ and $V(x)= \pm Z_{0}(\omega) I(x)$. The propagation coefficient $\gamma$ and line impedance $Z_{0}$ are given by

$$
\begin{aligned}
\gamma(\omega) & =\sqrt{i \omega C(i \omega L+R)} \text { and } \\
Z_{0}(\omega) & =\sqrt{\frac{i \omega L+R}{i \omega C}}
\end{aligned}
$$

To compute the frequency dependence of $R(\omega)$, the skin effect and the presence of the superconducting core have to be taken into account. In order to avoid a numerical computation of the fields inside the transmission line, we first consider a circular coaxial geometry with a composite inner conductor of outer radius $r_{i}$, which has a superconducting core of radius $r_{c} . r_{o}$ denotes the inner radius of the outer conductor. The dielectric between the conductors is assumed to have a homogeneous relative dielectric constant $\epsilon_{r}$. As the resistivity of the copper shield is much smaller than that of the wire, its resistance will be neglected for simplicity in the following. For this geometry, $C=2 \pi \epsilon_{r} \epsilon_{0} \log \left(r_{o} / r_{i}\right)$ and $L=2 \pi \mu_{0} \log \left(r_{o} / r_{i}\right)$. $R(\omega)$ is the ratio between the longitudinal electric field at the surface of the inner conductor, $E_{x}\left(r_{i}\right)$, and the total current $I$. Now $I=\oint \vec{B} \cdot d \vec{s}=2 \pi r_{i} B_{\phi}\left(r_{i}\right)$ and $E_{x}\left(r_{i}\right)=$ $\rho j_{x}\left(r_{i}\right)=\left(\rho / \mu_{0}\right)(\nabla \times \vec{B})_{x}=\left(\rho / \mu_{0} r\right) \partial\left(r B_{\phi}(r)\right) / \partial r$, so that we get:

$$
R(\omega)=\frac{\rho}{\mu_{0} 2 \pi r_{i}^{2}} \frac{\left.\frac{\partial}{\partial r}\right|_{r=r_{i}}\left(r B_{\phi}\right)}{B_{\phi}\left(r_{i}\right)} .
$$

To evaluate Eq. (3), we compute $B_{\phi}(r)$ as a solution of $\nabla^{2} B+2 i / \delta^{2} B=0$. In cylindrical coordinates with $\vec{B}=B_{\phi}(r) \hat{e}_{\phi}$, this equation reduces to

$$
\frac{\partial}{\partial r}\left(\frac{1}{r} \frac{\partial}{\partial r}(r B(r))\right)+2 i / \delta^{2} B(r)=0 .
$$

The solutions are first order Bessel functions of the first and second kind $J_{1}(\alpha r)$ and $Y_{1}(\alpha r)$ with $\alpha=-(1-$ $i) / \delta$. $Y_{1}$ diverges for $r \rightarrow 0$ while $J_{1}$ is regular there but diverges for $r \rightarrow \infty$. Thus the solution for a homogeneous resistive wire would be $B_{\phi}(r)=B_{\phi}\left(r_{i}\right) J_{1}(\alpha r) / J_{1}\left(\alpha r_{i}\right)$, For the wire with a superconducting core, the boundary condition at the interface is

$$
E_{x}\left(r_{c}\right)=\rho j_{x}\left(r_{c}\right)=\left.\frac{\rho}{\mu_{0} r} \frac{\partial}{\partial r}\right|_{r=r_{c}}\left(r B_{\phi}(r)\right)=0 .
$$

Together with a given value for $B_{\phi}\left(r_{i}\right)$, this determines the coefficients of the two solutions. To evaluate the radial derivative, it is useful to note that $\partial J_{1}(\alpha r) / \partial r=$ $(\alpha / 2)\left(J_{0}(\alpha r)-J_{2}(\alpha r)\right)$. The same holds for $Y_{1}$. Note that this procedure automatically takes the contribution of the magnetic field inside the resistive material to the total inductance into account. It is manifest in the imaginary part of $R(\omega)$.

The approximations made here are valid as long as the longitudinal electric fields in the dielectric are much smaller than the radial field and the radial current densities are much smaller than the longitudinal ones. The largest expected attenuation is on the order of $1 \mathrm{~dB} / \mathrm{cm}$ in the $\mathrm{GHz}$ range, so that the characteristic length scale over which the amplitude changes significantly along the propagation direction is about $10 \mathrm{~cm}$. Comparing this to the sub-mm radial length scale shows that the above condition holds well over the full frequency range under consideration.

To compute the $S$-matrix of a complete filter with capacitors to ground whose self resonance is modeled 
through a series inductance, we use the $A B C D$-matrix formalism. ${ }^{5,16}$ The result is compared to measured $S$ parameters in Sec. V.

\section{Effect of twisted pair geometry}

Due to the three-conductor geometry, there are two TEM modes. By symmetry, one is a common mode for which both wires have the same potential with respect to the shield, and the other one a differential mode with opposite voltages on each wire. Only the common mode can be measured using standard microwave equipment typically featuring coaxial connectors. In this case, the twisted pair can be treated as one single conductor.

Lacking exact knowledge of the cross section geometry, the capacitance and inductance per unit length, $C$ and $L$, cannot be calculated accurately and must be extracted from measurements. Thus, only the resistance $R(\omega)$ needs to be considered in order to transfer the model derived for coaxial lines to this common mode. At low frequencies, where $\delta \gg r_{i}$, Eq. (3) reduces to the DC resistance of the wire. A twisted pair without superconducting cores would trivially have half the resistance of a single wire. In the opposite limit $\left(\delta \ll r_{i}\right)$, the factor $2 \pi r_{i}$ in Eq. (3) must be replaced by the active circumference over which the wire surface is adjacent to the shield. The inner region, where the two wires face each other, will essentially be field free and thus contribute little to the conductance, leading to a correction factor slightly smaller than unity. Hence, one would expect the resistance of the pair to be up to a factor $1 / 2$ smaller than that of a single wire, with the largest corrections occurring at low frequency. We have incorporated this into the model by multiplying $R(\omega)$ as obtained from the known geometry and resistivity by a correction factor $\alpha$.

As only the common mode can be tested properly, the attenuation of the differential mode needs to be estimated. It is convenient to define all parameters for the differential mode with respect to the voltage between one wire and the ground shield. First, we consider $C$ and $L$ corresponding to this mode. If the symmetry plane between the two wires were replaced by a perfect conductor, the same boundary conditions would arise: by symmetry, the electric field must be normal to it and the magnetic field parallel. (The charge and current on the second wire can simply be considered as images of the first enforcing the boundary conditions on the hypothetical conductor.) Since the copper tape shield is also modeled as a perfect conductor, the differential mode should therefore have very similar parameters as the coaxial geometry. Now the capacitance $C$ is approximately proportional to the area over which the wire surface faces the ground conductor, while the inductance $L$ and resistance $R(\omega)$ are inversely proportional to it. (This is the origin of the factor $2 \pi r_{i}$ in equation 3 ). Thus, the product determining the propagation coefficient of the transmission line [Eq. (1)] remains unchanged compared to the common mode.
Only the line impedance $Z_{0}$ will be affected. A more intuitive explanation of this argument is that the common mode simply has a different effective area between the two conductors.

At frequencies where the discrete capacitors are important, the situation for the differential mode is slightly less favorable. As there is now only one rather than two parallel capacitors between the wire and shield, the capacitance seen by the propagating wave is simply half as large as for the common mode. The correction factor $\alpha$ on the other hand will be close to unity, but was larger than 0.5 for the common mode as explained above. Thus, one can expect the $R C$-time constant to decrease by about $20-30 \%$. Due to the increase of of $R(\omega)$ with higher frequency, the roll off frequency will change less than that.

\section{TEST RESULTS}

We tested a complete prototype filter and shorter copper tape worms without additional capacitors by soldering them to copper blocks fitted with SMA connectors. Both wires were connected to the center pin so that we measured the common mode transmission. The characterization from $50 \mathrm{MHz}$ to $20 \mathrm{GHz}$ was carried out using a HP 8510 vector network analyzer. Below 20 $\mathrm{MHz}$, we used a sine wave generator and a digital oscilloscope. All measurements were performed in a $50 \Omega$ environment. Additionally, we measured the capacitance per unit length at $\mathrm{kHz}$ frequencies using a capacitance bridge. For the common and differential modes, respectively, we obtained $0.61 \mathrm{nF} / \mathrm{m}$ and $0.23 \mathrm{nF} / \mathrm{m}$. The latter value was measured between the two wires with a floating shield so that it has to be doubled to conform with the definition form section IV C. The ratio of $0.61 / 0.46=$ 1.3 is just the ratio of the effective areas, corresponding to a correction $\alpha=1 / 1.3=0.77$ for the common mode resistance compared to the coaxial geometry.

Fig. 3 shows the result of the low frequency measurements at $300 \mathrm{~K}$ and $4 \mathrm{~K}$ and the corresponding models, for which we used $\alpha=0.65$. As expected, $\alpha=0.5$ slightly improves the agreement between model and data at low frequencies. The peak in the $4 \mathrm{~K}$ data around $3 \mathrm{MHz}$ is due to a resonance caused by the discrete capacitors and the inductance of the transmission line.

As can be seen in the inset of figure 3, measurements of the complete filter are not very revealing at higher frequencies because the signal disappears in the noise floor of the network analyzer. To extrapolate the filter performance, we measured two shorter tape worm pieces of length $l=52$ and $88 \mathrm{~cm}$ without additional capacitors (Fig. 4). Scaling the result to $l=4 \mathrm{~m}$, assuming that the attenuation is exponential in the lengths, shows that the two curves collapse reasonably well. The parameters for the model of a $4 \mathrm{~m}$ tape worm without capacitors were adjusted to obtain a good agreement with the data up to $1 \mathrm{GHz}$. The resulting capacitance of $C=0.55 \mathrm{nF} / \mathrm{m}$. 


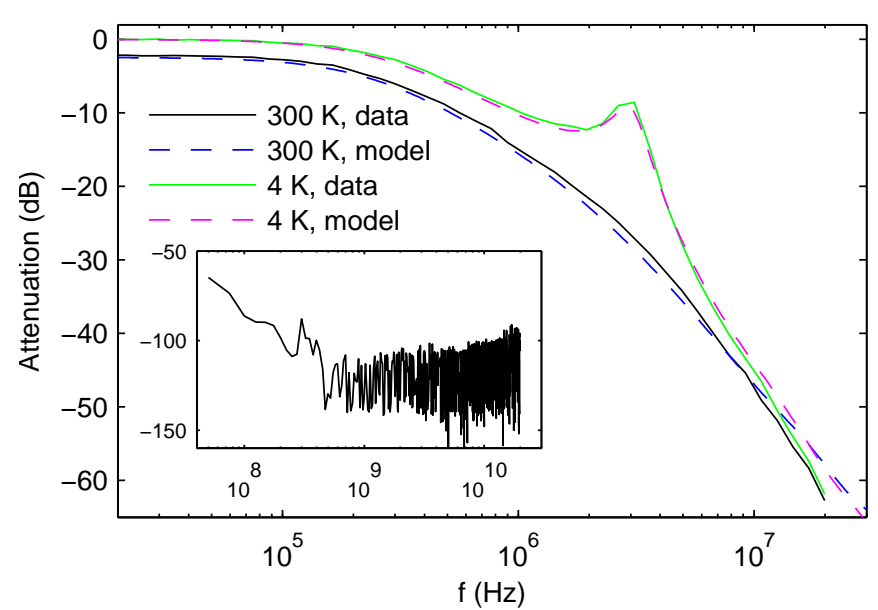

FIG. 3: (color online) Insertion loss of a filter prototype in a $50 \Omega$ line at low frequencies at $300 \mathrm{~K}$ and at $4 \mathrm{~K}$ with a superconducting core. Below the roll off, the $4 \mathrm{~K}$ data shows negligible dissipation. Above $10 \mathrm{MHz}$, the two measured curves converge. The peak in the $4 \mathrm{~K}$ data is due to an $L C$ resonance of the capacitors and the line inductance. The inset shows the attenuation from $50 \mathrm{MHz}$ to $20 \mathrm{GHz}$ at room temperature. Above about $500 \mathrm{MHz}$, the signal disappears in the noise floor of the network analyzer.

is in reasonable agreement with the value of $0.61 \mathrm{nF} / \mathrm{m}$ obtained from the bridge measurement. The ripple in the data at sub $\mathrm{GHz}$ frequencies is due to resonances in the transmission line caused by the impedance mismatch between the filter and the $50 \Omega$ environment. They can be fitted quite well with a model using the actual worm length, but they naturally do not appear in the model for $l=4 \mathrm{~m}$.

From the measured heat load due to a quasi-DC current running through a finished superconducting filter, we can set an upper limit of $3 \mathrm{~m} \Omega$ on its resistance, with a negligible increase of the dissipated power up to $1 \mathrm{kHz}$. Since this value includes solder joint resistances outside the filter, its actual resistance is most likely lower.

\section{CONCLUDING REMARKS}

There are a number of points that may have an effect on the filtering performance which we have not checked in detail, or which were changed after testing the prototype. Amongst those is the effect of using Silver epoxy rather than solder to connect wires and seal holes for epoxy injection. It is also likely that the RF tightness of unsoldered tape worm version is inferior compared to the one with the seam sealed by soldering. Due to aging of the tape adhesive and a resulting reduction of the capacitance between the wires and the shield, the filtering performance may degrade after thermal cycling. For the resistive version, we have relied on an adaptation of the

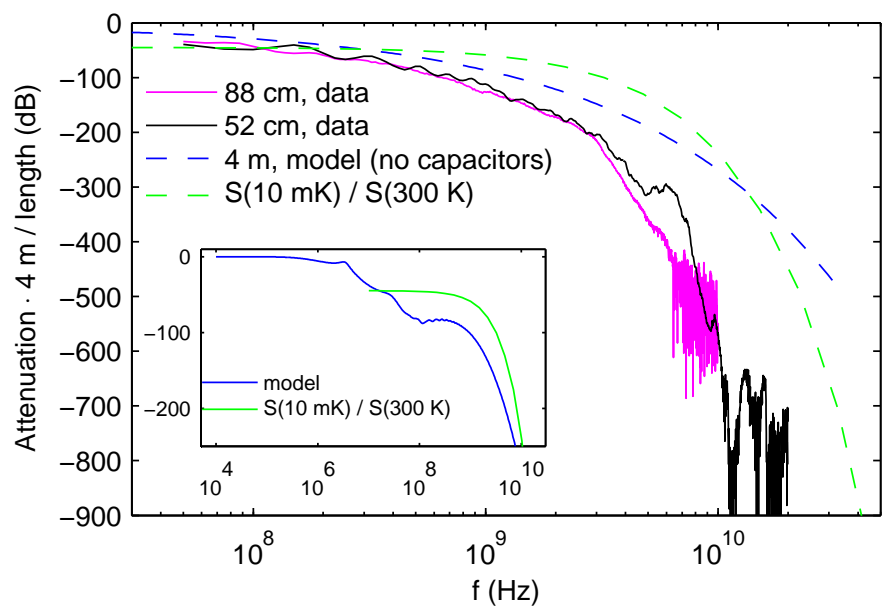

FIG. 4: (Color online) Insertion loss of short test pieces without discrete capacitors in a $50 \Omega$ line at room temperature. The data has been scaled to a total length of $4 \mathrm{~m}$ assuming that the attenuation (i.e. $\left|S_{21}\right|$ ) decays exponentially with increasing length. The data from two pieces of different length collapses well and shows a significantly higher attenuation than the model for a $4 \mathrm{~m}$ long line whose parameters have been chosen to match the scaled data below about $1 \mathrm{GHz}$. The inset shows the model for the complete filter using the parameters extracted from measurements. The ratio of the bosonic spectral densities at $300 \mathrm{~K}$ and $10 \mathrm{mK}$ for $f>10$ $\mathrm{MHz}$ is shown for comparison in both plots.

model from Sec. IV without extensive testing.

Although the design discussed here is seemingly simple, its development and implementation has taken several month of graduate student time, partly due to shorts in the first filter generation and the resulting need to modify the design. On the other hand, the construction of a complete filter was a matter of days once the procedures were finalized. Eliminating the capacitor boxes would make the implementation significantly simpler.

\section{Acknowledgments}

This work was supported primarily by the National Science Foundation under Grant No. DMR-0507931, and by the Department of Energy under Contract No. DEAC02-76SF00515. We would like to thank Ron Potok for useful discussion and Rob Schoelkopf for providing Ref. 5 before publication. 
Phys. 73, 2386 (1993).

2 J. M. Martinis and M. Nahum, Phys. Rev. B 48, 18316 (1993).

3 P. Bertet, I. Chiorescu, G. Burkard, K. Semba, C. J. P. M. Harmans, D. P. DiVincenzo, and J. E. Mooij, Phys. Rev. Lett. 95, 257002 (2005).

${ }^{4}$ K. Bladh, D. Gunnarsson, E. Hurfeld, S. Devi, C. Kristoffersson, B. Smalander, S. Pehrson, T. Claeson, P. Delsing, and M. Taslakov, Rev. Sci. Inst. 74, 1323 (2003).

${ }^{5}$ L. Spietz, J. Teufel, and R. J. Schoelkopf, condmat/0601316 (2006).

${ }^{6}$ P. G. Bjornsson, B. W. Gardner, J. R. Kirtley, and K. A. Moler, Rev. Sci. Inst. 72, 4153 (2001).

7 H. Bluhm, N. C. Koshnick, M. E. Huber, and K. A. Moler, Phys. Review. Lett. 97, 237002 (2006).

8 N. C. Koshnick, H. Bluhm, M. E. Huber, and K. A. Moler, Science 318, 1440 (2007).

9 B. L. T. Plourde, T. L. Robertson, P. A. Reichardt, T. Hime, S. Linzen, C. E. Wu, and J. Clarke, Phys. Rev. B 72, 60506 (2005).

10 J. M. Martinis, M. H. Devoret, and J. Clarke, Phys. Rev. B 35, 4682 (1987).
11 A. Fukushima, A. Sato, A. Iwasa, Y. Nakamura, T. Komatsuzaki, and Y. Sakamoto, IEEE Transactions on Instrumentation and Measurement 46, 289 (1997).

${ }^{12}$ F. P. Milliken, J. R. Rozen, G. A. Keefe, and R. H. Koch, Rev. Sci. Inst. 78, 24701 (2007).

13 A. B. Zorin, Rev. Sci. Inst. 66, 4296 (1995).

14 D. Vion, P. F. Orfila, P. Joyez, D. Esteve, and M. H. Devoret, J. of Appl. Phys. 77, 2519 (1995).

15 J. D. Jackson, Classical Electrodynamics, 3rd Ed. (USA : Wiley, 1998, 1998).

${ }^{16}$ D. M. Pozar, Microwave Engineering (USA: Addison Wesley, 1990, 1990).

17 This is valid in as long as the field penetrating the conductor does not contribute significantly to the inductance, which holds if $\delta \ll r_{o}-r_{i}$, i.e. above about $300 \mathrm{MHz}$ where $\delta=20 \mu \mathrm{m}$. At lower frequencies, about one skin depth of the resistive material acts as "dielectric" with infinite $\epsilon_{r}$. It contributes to $L$, but does not reduce $C$ below the value determined by the insulator because only magnetic, but no radial electric fields can penetrate the conductor. 